\title{
Traffic aware load balancing in AOMDV for mobile Ad-hoc networks
}

\author{
Gaurav Pathak ${ }^{1 *}$, Krishan Kumar ${ }^{2}$ \\ 1. Chandigarh University, Garuan, Punjab 140413, India \\ 2. Punjab University, Chandigarh 160014, India \\ *Corresponding author, Email: gauravpathak91@gmail.com
}

\begin{abstract}
The wireless networks have a very bright future in networks and communications because of which they have taken a high interest of the researchers. As the users increased the purpose to use MANETs, they also became more diverse and wide due to which better performance is needed in MANETs. QoS is needed for applications for an efficient communication and load balancing is a feature in the routing protocol that can help in a better use of the resources and can help to increase the performance of the network. We propose a new approach for load balancing in AOMDV routing protocol for MANETs that can enhance the network performance by selecting paths using the temporal load on the intermediate nodes and by distributing the load amongst the free nodes while transmission of data, which is proved by simulations in NS-2.
\end{abstract}

Keywords: AOMDV, NS-2, load balancing, QoS, wireless networks, MANETs

Citation: G. Pathak, K. Kumar. Traffic aware load balancing in AOMDV for mobile Ad-hoc networks [J]. Journal of communications and information networks, 2017, 2(3): 123-130.

\section{Introduction}

MANET (Mobile Ad-hoc Network) is a collection of mobile devices and is self configuring, dynamically changing, multi-hop wireless network which forms a communication network via multi hop wireless network connection. It is a self-organizing network, without any central control. Nodes in the network communicate with another node only if it lies within its transmission range. Mobile Ad-hoc networks have potential applications in civilian and military environments. The dynamic changes in the topology of MANET make routing a challenging task, as the existing path is rendered inefficient and infeasible. The major issues for mobile Ad-hoc networks are MAC (Medium Access Control), routing, security and QoS (Quality of Service) provisioning ${ }^{[1]}$, which is mainly because of node mobility, link failure, limited available bandwidth. The routing challenge in MANETs is to provide an optimal path in less amount of time for data forwarding with limited power supply, processing and memory resources ${ }^{[2]}$.

The problem in existing routing protocols is that there is no fair distribution of the traffic amongst the paths in the network the nodes that lie in more dense areas are used more by different paths and hence are exhausted and that leads to degradation in the performance of the routing protocol. A load balancing feature needs to be added in the existing protocols to distribute the workload fairly in the network.

Using multipath routing protocols like AOMDV can help in keeping backup routes in case of a route breakage but still they are not providing any load balancing feature because in case of less mobile con- 
ditions when route breakage is not there or less, it uses only the primary path for the purpose of data and other routes are not used ${ }^{[3]}$.

\section{Routing in MANETs}

Routing is the mechanism of forwarding packet towards its destination using most efficient path.

In MANET routing is classified into two types according to availability of route:

1. Pro-active routing: Nodes in a wireless Ad-hoc network should keep track of routes to all possible destinations so that when a packet needs to be forwarded, the route is already known and can be used immediately.

2. Re-active routing: When a node wants to send packets to a destination but has no routes to the destination, it initiates a route discovery process within the network. Once a route is established, it is maintained by a route maintenance procedure until the destination becomes inaccessible or until the route is no longer needed.

\section{AOMDV (Ad-hoc on Demand Mul- tipath Distance Vector)}

AOMDV shares various characteristics with AODV. It is a distance vector based routing protocol. It is an on-demand routing protocol. The difference lies in the number of routes in each route discovery. In AOMDV, RREQ propagation from the source towards the destination establishes multiple reverse paths both at intermediate nodes as well as the destination. Multiple RREPs traverse these reverse paths to form multiple forward paths to the destination and intermediate nodes. The main focus of AOMDV is to find multiple paths to the destination which are loop free and are disjoint in nature. AOMDV can find two types of disjoints in the network "node disjoint" and "link disjoint", in node disjoint paths, no two paths for same destination can have a common intermediate node. Similarly in link disjoint paths, no two paths for same destination can have a common link in between the path. AOMDV relies on routing information as much as possible as of AODV for routing purpose. It does not use any extra control packets to find multiple paths to the destination i.e. no extra overhead is added ${ }^{[3]}$. In Fig. 1, the destination variable is for the destination address of the path. Sequence number is used to tell the freshness of the path, higher the sequence number fresher is the path. Advertised hop count is the maximum hop count used in the previous paths that existed for that destination. In the route list the next hop is the address of the next node from the current node, last hop is the node before the destination, hop count carries the number of nodes in that path and timeout is used to tell the lifetime of the path.

\section{Related work}

There have been many proposals for the purpose of load balancing in routing of MANETs.

Lee et al. in Ref. [4] proposed DLAR (Dynamic Load Aware Routing) protocol which considers the load of the intermediate nodes as the main criteria of selecting the routes and also monitors the congestion status of active routes for the purpose of path reconstruction. In DLAR the nodes piggyback their load information in RREQ and then broadcast it further to its neighbours. When destination receives the RREQ it waits for a while to receive maximum RREQs from different nodes, then it chooses the least loaded path between them.

Tekaya et al. ${ }^{[5]}$ presented a new multipath QoS routing with load balancing. There are two main proposals in this work. One is load balancing mechanism to fairly distribute the traffic on different active routes, the other is route discovery mechanism based on QoS parameter such as delay and throughput. Firstly, they proposed a new multipath routing protocol called LB-AOMDV with a new metric which is the buffer size of the less congested routes. Then they add QoS to their proposal LB-AOMDV protocol which includes delay and throughput parameters. It takes the advantage of the RREQ message to exchange the essential information to achieve the QoS requirements. Enabling a QoS constrained 


\begin{tabular}{|c|c|c|c|c|c|c|}
\hline destination & sequence number & advertised hop count & \multicolumn{4}{|c|}{ route list } \\
\hline & & & $\begin{array}{c}\text { next }^{\cdot} \text { hop }_{1} \\
\text { next } \text { hop }_{2} \\
\ldots\end{array}$ & $\begin{array}{c}\text { last 'hop } 1 \\
\text { last }{ }^{\prime} \text { hop }_{2} \\
\ldots\end{array}$ & $\begin{array}{c}\text { hop }^{\text {count }} \\
\text { hop } \text { count }_{2} \\
\ldots\end{array}$ & $\begin{array}{c}\text { timeout }_{1} \\
\text { timeout }_{2} \\
\ldots\end{array}$ \\
\hline
\end{tabular}

Figure 1 Routing table entry in $\mathrm{AOMDV}^{[3]}$

from source to destination is acquired in new protocol QLB-AOMDV.

Tarique et al. ${ }^{[6]}$ presented a survey of most recent multipath routing protocols for MANETs. The surveyed protocols showed that multipath routing can improve network performance in terms of delay, throughput, reliability and lifetime. Yet it is hard to find a single protocol or a set of protocols that can improve all these performance parameters. Selection of a multipath routing protocol depends on a particular application and trade-offs. Some of the objectives here are energy efficiency, low overhead, reliability and scalability.

Soundararajan et al. ${ }^{[7]}$ presented a new approach MLBRBCC (Multipath Load Balancing and Rate Based Congestion Control) based on rate control mechanism for avoiding congestion in network communication flows, in which multi-path routing can balance the load better than the single path routing in Ad-hoc networks, thereby reducing the congestion by dividing the traffic in several paths. They proposed an approach that contains an adaptive rate control based technique in which the destination node copies the estimated rate from the intermediate nodes and the feedback is forwarded to the sender through an acknowledgement packet. Since the sending rate is adjusted based on the estimated rate, this technique is better than the traditional congestion control technique.

Li et al. ${ }^{[8]}$ proposed NDMR to overcome the shortcomings of unipath routing protocols. NDMR has two novel aspects by which it reduces routing overhead dramatically and achieves multiple nodedisjoint routing paths. It is evident from simulation results that NDMR outperforms both AODV and DSR because multiple node disjoint routing paths provide robustness to mobility. This protocol is best suitable for small network and not more efficient in large network due to control overhead in the reply packet.

Gulati et al. ${ }^{[9]}$ surveyed different types of QoS routing protocol. The contributions of the paper are as follows: The basic concepts and challenges of QoS routing in MANETs have been reviewed. The classification of the protocols has been done on the basis of multi-path, cross layer, stability, bandwidth reservation, load balancing and power efficiency based approaches. The protocols are selected in such a way so as to highlight many different approaches to QoS routing in MANETs, while simultaneously covering most of the important advances in the field. For each protocol, the functionality and main features are described briefly. The strengths and weaknesses of these protocols have also been provided and, finally, a comparison of all the QoS routing protocols has been done so as to explore the future areas of research. All the QoS routing protocols discussed can further be explored in the areas of bandwidth estimation, route discovery, resource reservation, route maintenance and cross-layer design to improve their performance. Many areas of research in this field provide considerable challenge and potential to enhance the growth and proliferation of MANETs and their applications. These areas include power consumption, resource availability, location management, inter-layer integration of QoS services, support for heterogeneous MANETs as well as stability, robustness and security. Effective and efficient solutions to these issues require the design and development of new QoS routing protocols in MANETs. So, on the basis of survey we choose multipath routing approaches to provide QoS in MANET because in multipath routing approaches the protocol provides bandwidth aggregation, minimizing end-to-end delay, increasing fault tolerance, enhancing reliability and load balancing. On the 
basis of these features we try to provide QoS in MANET.

Gupta et al. ${ }^{[10]}$ proposed a rate based congestion management technique, in this technique the sender monitors the delay in acknowledgments from te destinations to measure the congestion in the network, and then accordingly take the decision to change the data rate in the network, which helps in keeping the congestion level of network in check.

Deshmuk et al. ${ }^{[11]}$ proposed an energy based load balancing scheme in which the paths are chosen according to the total energy available in the nodes, existing in the paths. The path with the maximum energy in the intermediate nodes is chosen to transmit the data. This helps in maintaining the energy level of the nodes in the network.

Tab. 1 shows the comparison of different multipath routing protocols in MANETs based on their properties.

Table 1 Comparison of multipath routing protocols

\begin{tabular}{lccc}
\hline & AOMDV & MSR & AODVM \\
\hline 1. routing category & reactive & reactive & reactive \\
2. loop free paths & no & no & no \\
$\begin{array}{l}\text { 3. routing overhead } \\
\text { control }\end{array}$ & no & yes & yes \\
$\begin{array}{l}\text { 4. node-disjoint paths } \\
\text { 5. complete routes }\end{array}$ & no & yes & no \\
$\begin{array}{l}\text { known at source } \\
\text { TTL limitation }\end{array}$ & no & yes & yes \\
7. QoS support & no & no & no \\
8. multicast support & no & no & no \\
9. power management & no & no & no \\
10. security support & no & no & no \\
\hline
\end{tabular}

\section{Proposed methodology}

Although AOMDV saves multiple paths for the purpose of data transmission but it uses only one path till it is valid and keeps others as a backup. This can lead to high overhead on a single path and waste of resources in case the primary path does not break and the other paths will never be used if primary path stays valid.
To overcome this problem we propose an approach TALB-AOMDV (Traffic Aware Load Balanced AOMDV) the proposed approach considers queue length along with the hop count as a metric for path selection and after path selection the data is equally distributed along the paths to divide the load amongst the path so that biasness could be removed in usage of the path in the network. The queue length field is added in RREQ packet, which carries the total queue length of the path along with which it has travelled. The path is selected according to the buffer size of the request.

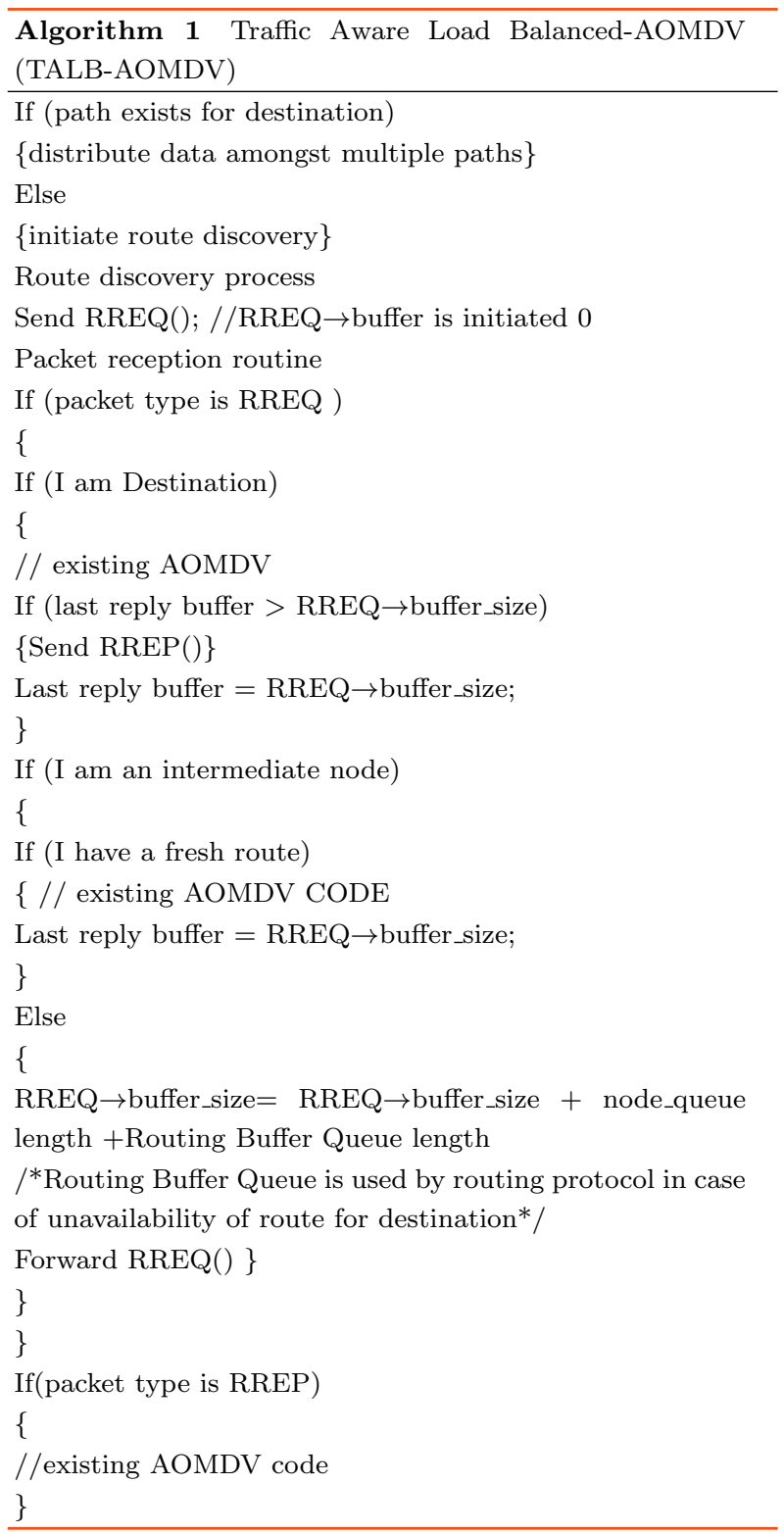


Algorithm 1 explains that the node sends the RREQ packet when it needs to transmit data and it doesnt have a route to destination and source initiates the buffer_size of RREQ to zero, when a node receives RREQ, if it has a fresh route it sends reply to the source otherwise it adds its routing buffer size and queue length to RREQ buffer_size and forwards it. If the node is destination it sends RREP and stores the RREQ buffer_size. Now it sends the next reply to same source only if RREQ buffer_size is less than the last RREQ buffer_size for which reply was sent.

\section{$6 \quad$ Simulations and results}

A detailed simulation model is used which is based on NS2. DCF (Distributed Coordination Function) on 802.11 is used as MAC layer Protocol. DCF of 802.11 uses RTS (Request to Send) and CTS (Clear to Send) for unicast data transmission to the neighbor nodes. The RTS/CTS exchange precedes the data transmission and performs virtual sensing and medium reservation to reduce the problem of hidden terminal in wireless networks. CSMA/CA is used to transmit the data through medium. WaveLAN is modelled as shared media with nominal bit rate of $2 \mathrm{Mbit} / \mathrm{s}$ with radio range of $250 \mathrm{~m}$. CBR (Continous Bit Rate) traffic sources are used. The source destination pairs are spread randomly over the network. 512 bytes data packets are used. The mobility model used is random waypoint in a rectangular area of $1000 \times 1000$ with 50 nodes. The node moves with a fixed mobility towards a destination where it stops for a period of time (pause time) then moves further to the next destination. The simulation run is for 200 simulated seconds. The detailed description of the simulation scenario is given in Tab. 2 .

Performance metrics:

1) Packet delivery ratio. The packet delivery ratio in this simulation is defined as the ratio between the number of packets sent from constant bit rate sources (CBR, application layer) and the number of receiving packets by the $\mathrm{CBR}$ sink at destination. It specifies the packet loss rate, which limits the maximum throughput of the network.

2) End to end delay. This metric represents an average end-to-end delay and indicates how long it took for a packet to travel from the source to the application layer of the destination.

3) Throughput. Throughput is total packets successfully delivered to individual destinations over total time.

Table 2 Parameters used in simulation

\begin{tabular}{cc}
\hline parameter & value \\
\hline dimension & $1000 \times 1000(m \times m)$ \\
number of nodes & 50 \\
simulation time & $200 \mathrm{~s}$ \\
traffic type & $\mathrm{CBR}$ \\
number of connections & $5-35$ (variable) \\
packet size & 512 bytes \\
MAC layer & IEEE $802.11 \mathrm{~b}$ \\
buffer size & 50,75 \\
propagation radio model & two ray ground \\
physical layer & bandwidth $2 \mathrm{Mbit} / \mathrm{s}$ \\
pause time & 20 \\
rate & 10 packet $/ \mathrm{s}$ \\
\hline
\end{tabular}

The comparison of existing AOMDV and the proposed approach TALB-AOMDV is done. TALBAOMDV and AOMDV both are compared in different scenario with variation of number of mobile connections between the nodes in the network. An overhead is added because of the addition of a new field in RREQ packet but it can be tolerated because we use paths with minimum load and the packet lost in the network will get decreased, and as the field is only added in route request packet which is issued only when route discovery is required,so, it will not have much of a negative effect on the network performance as the frequency of route request packet is not very high in the network unless the path breakage in the network is very high which can occur only because of very high mobility in the network.

In Fig. 2(a) the end to end delay of TALBAOMDV comes out to be less than AOMDV because we are using the paths which are having fewer 
loads or lesser number of packets in the queue. Due to which the queue delay decreases in the path and hence the total delay comes out to be less than AOMDV. In Fig. 2(b) The packet delivery ratio is

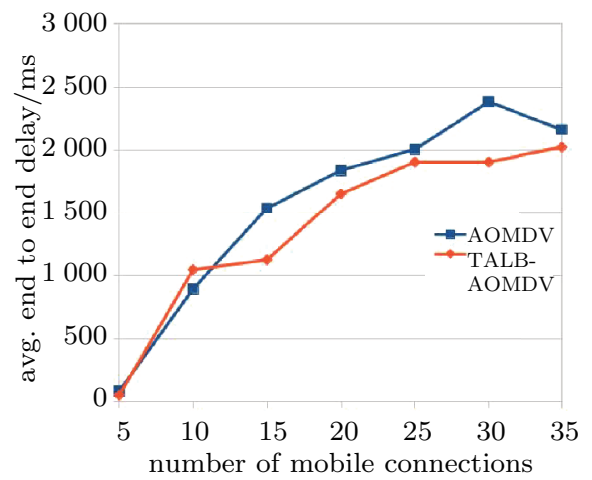

(a)

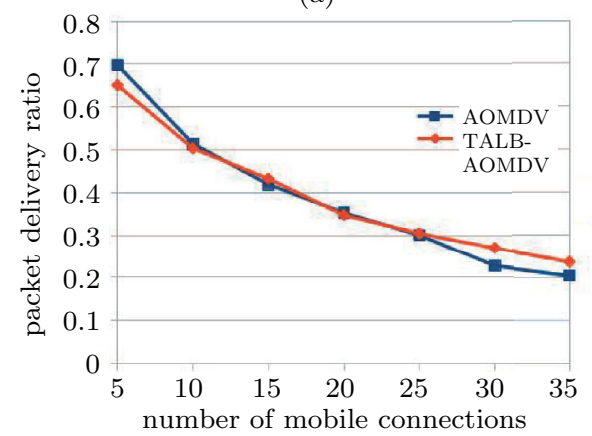

(b)

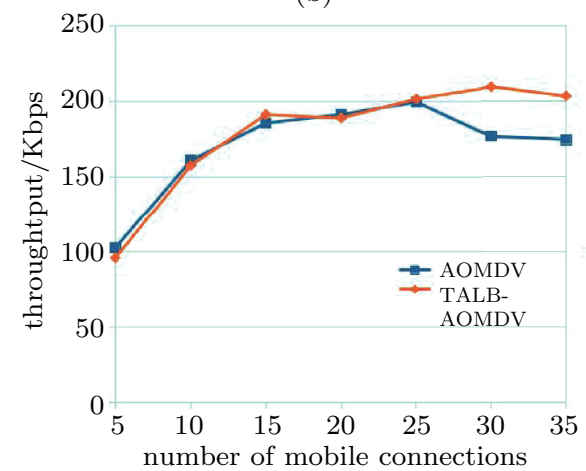

(c)

Figure 2 Performance on queue length 50 of TALBAOMDV and AOMDV: (a) avg. end to end delay; (b) packet delivery ratio;(c) throughput

approximately same when using paths with low load in the network but as the load I in the network is increased the performance of AOMDV decreases and TALB-AOMDV takes lead because when the load in low in the network the network load balancing is not much needed in the network as the resources in the network fulfill the need of the communications, but as the load in the network increases and one single path has not enough resources for communication then comes the need of load balancing in the network. TALB-AOMDV distributes the traffic to different paths which are chosen according to the least buffer size to divide the load in the network; this increases the packet delivery of the network. In Fig. 2(c) the throughput of the network of both AOMDV and TALB-AOMDV are approximately same in the network but as the load increases in the network the throughput decreases because high load leads to congestion and due to congestion packets are not received by destinations, as they are dropped by the intermediate nodes due to queue overflow. So when we are using a single path for transmission the probability of packet drop is high when congestion occur in that path, that is why the throughput of AOMDV drops, on the other hand TALB-AOMDV has a better throughput, the reason is that TALB-AOMDV uses multiple paths and distributes the traffic, which decreases the probability of packet drops.

Fig. 3(a) shows the results of end to end delay in comparison of AOMDV and TALB-AOMDV on interface queue length 75 . When queue length is increased the end to end delay also increases as only delivered packets are considered in end to end delay and the queue delay of these delivered packets increases due to increase in queue length. Fig. 3(b) shows the throughput results at queue length 75 . The throughput and packet delivery ratio increases as we are using less loaded paths whose queues are having less number of packets, so the packet drops because of queue overflow are less than that of normal AOMDV which results in a better throughput and packet delivery in the network. Fig. 3(c) illustrates the comparison of AOMDV and TALB-AOMDV with respect to packet deliver ratio when queue length is taken 75 , the packet delivery ratio comes out to be better when queue length is increased as more packets can be buffered in queue and as the figure shows that packet delivery ratio of TALB-AOMDV, it takes 
the lead from the second value which was not the case when queue length was taken 50 . It is because when queue length is increased and the packets are distributed the chances of packet drop are even lesser at the intermediate node as the congestion will be avoided until queue is full and longer the queue size more will be the packet delivery ratio, but queue length cannot be taken too high as it increases the overhead on the node.

The main benefit of the proposed scheme is that the protocol is able to provide reasonable amount of better average end to end delay and there is no tradeoff between any other QoS metric.

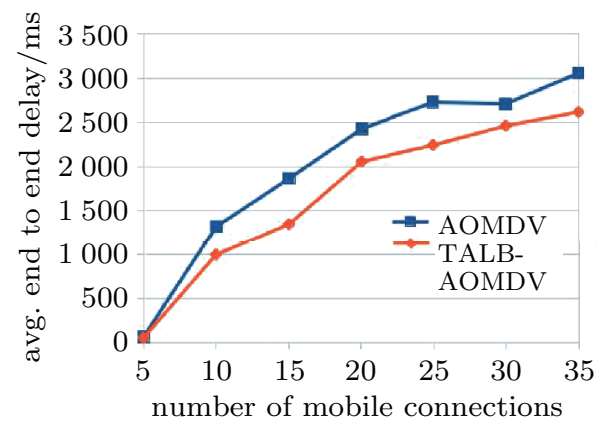

(a)

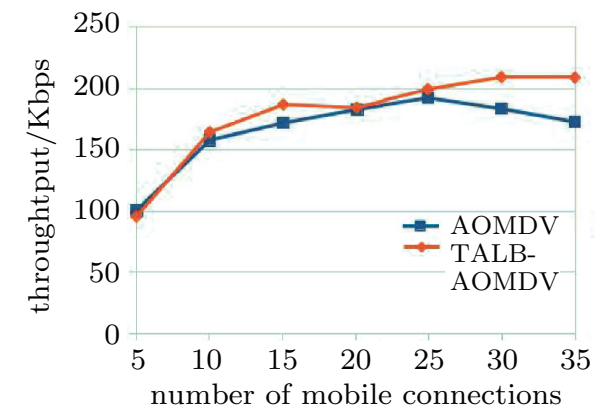

(b)

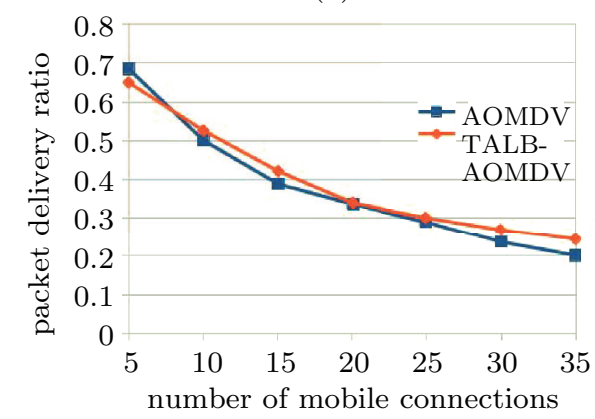

(c)

Figure 3 Performance on queue length 75 of TALBAOMDV and AOMDV: (a) avg. end to end delay; (b) throughput; (c) packet delivery ratio

\section{Conclusion}

The proposed approach performs better than the existing AOMDV protocol in higher loads as AOMDV only uses one path at a time. On the other hand, our protocol TALB-AOMDV distributes the traffic amongst various paths which helps in distributing the load amongst more nodes and hence leads to better resource utilization which ultimately leads to more network lifetime and balanced power consumption.

\section{Future work}

The proposed approach performs better than AOMDV but can even perform better by using regular updates on the buffer information of the nodes in the paths so that the nodes can take dynamic decisions regarding using better routes in the middle of data transmission which can lead to even better use of resources of the network. If the energy will also be used as a path selection metric the proposed scheme can also help in increasing the network lifetime.

\section{References}

[1] Internet Engineering Task Force (IETF). Mobile Ad-hoc Networks (MANETs) Working group charter [EB/OL]. http://www.ietf.org/html.charters/manetcharter.html.

[2] M. Tekaya, N. Tabbane, S. Tabbane. Multipath routing with load balancing and QoS in Ad-hoc network [J]. International journal of computer science and network security, 2010, 10(8), 280-286.

[3] M. K. Marina, S. R. Das. On-demand multipath distance vector routing in Ad-hoc networks [C]//The 9th International Conference on Network Protocols, Riverside, USA, 2001: 14-23.

[4] S. J. Lee, M. Gerla. Dynamic load-aware routing in Adhoc networks [C]//IEEE International Conference on Communications, Helsinki, Finland, 2001: 3206-3210.

[5] M. Tekaya, N. Tabbane, S. Tabbane. Multipath routing mechanism with load balancing in Ad-hoc network [C]//International Conference on Computer Engineering and Systems (ICCES), Cairo, Egypt, 2010: 67-72.

[6] M. Tarique, K. E. Tepe, S. Adibi, et al. Survey of multipath routing protocols for mobile Ad-hoc networks [J]. Journal of network and computer applications, 2009, 32(6): 1125-1143. 
[7] S. Soundararajan, R. S. Bhuvaneswaran. Multipath load balancing \& rate based congestion control for mobile Ad-hoc networks (MANET) [C]//The 2nd International Conference on Digital Information and Communication Technology and its Applications (DICTAP), Bangkok, Thailand, 2012: 30-35.

[8] X. F. Li, L. Cuthbert. On-demand node-disjoint multipath routing in wireless Ad-hoc networks [C]//The 29th Annual IEEE International Conference on Local Computer Networks, Tampa, USA, 2004: 419-420.

[9] M. K. Gulati, K. Kumar. QoS routing protocols for mobile Ad-hoc networks: a survey [J]. International journal of wireless and mobile computing, 2012, 5(2): 107-118.

[10] S. Gupta, G. Prasad. Enhanced load balancing and delay constraint AOMDV routing in MANET $[\mathrm{C}] / /$ Symposium on Colossal Data Analysis and Networking (CDAN), Indore, India, 2016: 1-6.

[11] S. R. Deshmukh, V. T. Raisinghani. EALBM: energy aware load balancing multipath routing protocol for MANETs [C]//The 11th International Conference on Wireless and Optical Communications Networks (WOCN), Vijayawada, India, 2014: 1-7.

\section{About the authors}

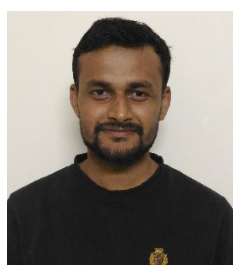

Gaurav Pathak [corresponding author] has completed his B.Tech. in computer science and engineering from Lovely Professional University, Phagwara, India and his M.Tech. in computer science and engineering from Punjab Institute of Technology Kapurthala, Punjab, India, and has worked in Chandigarh University as assistant professor in Department of Computer Science. (Email: gauravpathak91@gmail.com)

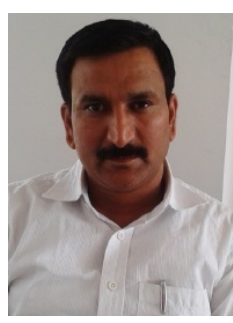

Krishan Kumar has done B.Tech. computer science and engineering from NIT Hamirpur in 1995. He finished his M.S. software system from BITS Pilani in 2001. He finished his Ph.D. from Department of Electronic and Computer Engineering IIT Roorkee in 2008. Currently he is professor in Department of Information Technology Puinjab University, Chandigarh, India. (Email: k.salujasbs@gmail.com) 